\section{Education and the Care of Older People: Is This the Time for Renewal?}

\author{
Isabel Higgins \\ School of Nursing and Midwifery, University of Newcastle, Callaghan, Australia
}

The final report of the royal commission into aged care in Australia, "Care, Dignity and Respect” (Royal Commission into Aged Care Quality and Safety, 2021), was handed down in February this year and tabled for public viewing. Major findings indicate a lack of funding for care and support for older people leading to poor outcomes in residential, home and community care settings. Staffing for services, clinical care and the support of family/carers has been a major concern in Australia for many years with understaffing and widespread low levels of education amongst staff including nurses, personal carers and support staff. Importantly, attempts to improve help, care and support in home settings guided by an international best practice model, consumer directed care (CDC) (Ottman, Allen \& Feldman, 2013; Ottman \& Mohebbi, 2014) has led to fewer hours of care per day than would be otherwise provided in other care settings.

Older people in need of care and support in any setting rarely need one form of care or support. As noted by the commissioners (2021), ageing brings with it physiological and sociological decline which in the presence of chronic co-morbidities renders a range of challenges for the older person. As physiological decline can be anticipated, so too should emotional, psychological and social aspects of life. Support and care for older people requires maintaining a level of social functionality in order to support emotional and psychological health. However, in the light of a range of complex and chronic co morbidities, health expertise is essential to focus healthy ageing with preservation of ability, disease prevention and/or mitigation and appropriate end of life care.

The CDC model of care mandates meaningful relationships with older people and skilled care to ensure safety, quality of care and support, and dignity and respect for individuals. Skillful care and support helps to maintain functionality for daily living, mitigates disease progression, and deterioration towards frailty and when needed it ensures quality at the end of life. In the absence of meaningful relationship-based encounters with health professionals and carers and a CDC approach to care, any form of support is merely a transaction of deed falling short of quality and care. The authors of the royal commission in Australia argue persuasively using many exemplars that care of older people and services are substandard in all care settings. Of concern is that these claims reflect the value placed on older people in our society.

Contemporary care of older people is mainstream care across all settings: acute, aged, community and home. However many nurses, health professionals and carers are inadequately prepared educationally to provide care and support for people who are experiencing physiological decline associated with ageing, co morbidities and decline in the ability to adapt to many life stressors. Older people, regardless of the setting or context for care require nurses and health professionals with a suite of skills to manage older people with complex intersecting health breakdown which is often confounded by polypharmacy, psychological and social concerns and a range of personal support needs. Responding to these needs and life preferences requires an holistic approach to managing and supporting changing circumstances, as well as skilled, in-

\section{Editorial}

pISSN 2288-8675 · eISSN 2508-9145

J Probl Based Learn 2021;8(2):51-52

https://doi.org/10.24313/jpbl.2021.00094

Received: October 13, 2021

Accepted: October 14, 2021

Corresponding author:

Isabel Higgins

School of Nursing and Midwifery,

University of Newcastle, 66 Rickard

Street, Bateau Bay, NSW 2261,

Australia

Tel: +61-437-397 610

E-mail: Isabel.higgins@newcastle. edu.au

(C) 2021 International Society for Problem-Based Learning

(c) This is an Open Access article distributed under the terms of the Creative Commons Attribution Non-Commercial License (http://creativecommons.org/licenses/ by-nc/4.0/) which permits unrestricted non-commercial use, distribution, and reproduction in any medium, provided the original work is properly cited. 
tuitive observation, communication, care, and flexibility. It requires professionals to provide leadership management and clinical supervision, to model the expectations for care, dignity and respect; to strongly advocate for the older person and his or her loved ones, and to ensure the quality standards, continuity and integration of care at all points in their life journey. It requires care and support staff to be sensitive to the needs of older people, to observe, to respond appropriately and communicate with the team of professionals and carers clearly on all matters of transaction and to act with integrity.

Professional nursing practice around the world is guided by practice standards and codes of ethics and professional conduct. Nurses, leaders in societies around the world, are advocates for people across age groups and the generations. They have a professional mandate to ensure the best possible care and support is available to all older people in any care setting. They have a mandate for humanity. Paving the way for all older people regardless of the context for care demands a multi focused educational approach: ensuring nurses are equipped with research knowledge and the best available evidence, skilled for leadership, assertiveness and advocacy, open to creativity, innovation and ongoing research. Expert care mandates the use of evidence and the application of theory in clinical practice situations. It requires the application of human and nursing sciences and the exploration and use of models and frameworks to ensure the best possible delivery of care for Quality. Quality and care afford dignity and respect and this, unequivocally, is international best practice. Above all, older people need to be the focus of learning stimuli in a range of contextual, social and cultural settings.

Readers of this journal may consider that whilst Australia is but one of many nations and that its care and systems of care of older people is both like and unlike other nations, what needs to be heeded is how easily older people can become marginalized in any society and how frailty exacerbates marginalization. Older people become objectified as a problem to be solved or a problem that is overlooked or forgotten with transactions of care falling short of quality. Problem based learning (PBL) approaches require real world learning situations generated from across settings, contexts, and cultures. The demands for better care for older people around the world are replete with exemplars for learning, of situations in need of improvement and critical analysis, of research problems that need to be investigated, tested and resolved, of the need for innovation and creativity, communication, teamwork efforts and demonstrations of humanity.

Undergraduate and post graduate studies are both important platforms for improving the care and support for older people and for more informed leadership across all settings for care. However, all levels of carer also need contemporary and ongoing education and certification using a PBL approach. Ultimately education and ongoing learning using PBL is essential for all health professionals and workers in order to appreciate the complexities of care and support needs and to ensure quality and care for older people around the world.

\section{CONFLICT OF INTEREST}

The authors declared no conflict of interest.

\section{REFERENCES}

Ottmann, G., \& Mohebbi, M. (2014). Self-directed community services for older Australians: a stepped capacity-building approach. Health \& Social Care in the Community, 22, 598-611.

Ottmann, G., \& Allen, J. (2013). A systematic narrative review of consumer-directed care for older people: implications for model development. Health \& Social Care in the Community, 21, 563-581.

Commonwealth of Australia Royal Commission into Quality and safety. (2021). Retrieved Month Date, Year, from https://agedcare.royalcommission.gov.au/publications. 